\title{
Review of Sodium Valproate Clinical and Biochemical Properties
}

\author{
Zahra Tolou-Ghamari ${ }^{1, *} ;$ Abbas Ali Palizban ${ }^{2}$ \\ ${ }_{1}^{1}$ Isfahan Neurosciences Research Centre, Isfahan University of Medical Sciences, Isfahan, IR Iran \\ ${ }^{2}$ Department of Clinical Biochemistry, Faculty of Pharmacy, Isfahan University of Medical Sciences, Isfahan, IR Iran \\ *Corresponding author:Zahra Tolou-Ghamari, Isfahan Neurosciences Research Centre, Isfahan University of Medical Sciences, Isfahan, IR Iran. E-mail: toloeghamari@pharm.mui.ac.ir
}

Received: April 23, 2014; Accepted: June 10, 2014

\begin{abstract}
Valproic acid is a commonly used anti-epileptic drug (AED) for prescription to control convulsion attacks. The aim of this study was to providea review of drug managementamong adultepileptic population. Topic words that searched were "Valproic acid,Pharmacokinetics, Epilepsy and Iran" that search in DOAJ, Google Scholar, PubMed and Web of Science. A total of approximately 1200 articles were found and at the end, 41 articles were reviewed. With an oral dose of $10-15 \mathrm{mg} / \mathrm{kg} /$ day in three divided doses, target blood level ranges from $50-100 \mathrm{mg} / \mathrm{L}$. With a half-life of 9 - 16 hours, it seems that the valproic acid has a variable absorption phase. The pattern of metabolic pathways seems to be through the glucuronide conjugation and mitochondrial $\beta$-oxidation. The drug causes delay in carbamazepine elimination and may increase the amount of its metabolites. Valproic acid could decrease the clearance of amitriptyline and nortriptyline. Aspirin increase its' trough level. The doses of olanzapine suggested to be reduced when co-medicated with valproic acid. To specify the amount and effects of administrated dose an attentive pharmacotherapy-guideline seems to be useful. Due to CYP450 dependent metabolic pathways (inhibition or induction), therapeutic drug monitoring (TDM) in simultaneous prescription of valproic acid with carbamazepine, lamotrigine, phenytoin, topiramate, amitriptyline, nortriptyline, aspirin, meropenem, olanzapine and clonazepam, manifested to be necessary in clinical practice.
\end{abstract}

Keywords: Valproic acid; Epilepsy; Pharmacokinetics; Clinical properties; Combination therapy

\section{Context}

Burton in 1882 was found an analogue of valeric acid with two propyl groups called valproic acid(VPA) [1]. Indeed VPA is a carboxylic acid, which is a clear liquid at ambient temperature. Eighty years later the anti-epileptic properties of this drug have been recognized and then again five years later it has been approved as an anti-epileptic drug (AED). In fact it was accepted by the US Food and Drug Administration in 1978 for management of some kind of epileptic seizures such as complex partial seizures and simple and complex absence seizures. An equimolar mixture of VPA and sodium valproate called divalproate, has been accessible in the United States since at 1983. The other brand names are Depakene, Depakote, Depakote ER. Its' international brand name called also Depakine. Two forms of drugs have extra FDA approvals. The extended-release form of drug (Depakote ER) is designated for migraine headache prophylaxis and the delayed-release product (Depakote) carries an indication for the short term management of acute manic episodes associated with bipolar disorder [1-4].

As shown in Figure 1, VPA has a different mechanism of actions. In bipolar disorder it effect by inhibiting gammaaminobutyric acid (GABA) transaminase and then increases the amount of GABA within brain. However, several other mechanisms of action have been proposed for this AED in recent years. The drug could be categorized as a broad-spectrum AED, because it suppress both sodium and calcium channels. It is a histone deacetylase inhibitor. Sodium valproate possibly could affect sodium channels and potentiate GABA receptors. It also increases the formation of GABA [5]. It is well known that VPA belongs to group of drugs with narrow therapeutic window and as prescription of VPA in Iranian epileptic patients continues to increase [4-6], an approach adjoining clinical pharmacotherapy guideline to control seizure attack was of interest that reviewed.

\section{Evidence Acquisition}

Data of this review were collected from the authors clinical experiences' related to pharmacological, biochemical inter and intra individual management of antiepileptic drugs in patients with epilepsy [5-7]. Directory of Open Access Journals (DOAJ), Google Scholar, PubMed (NLM), LISTA (EBSCO), Web of Science were searched. The key words were relevant to Valproic acid, Pharmacokinetics, Epilepsy and Iran. A total of approximately 1200 articles were found. In next step approximately a total of 41 articles relevant to the clinical properties of valproic acid were selected and reviewed correspondingly.

Copyright (c) 2015, Zahedan University of Medical Sciences. This is an open-access article distributed under the terms of the Creative Commons Attribution-NonCommercial 4.0 International License (http://creativecommons.org/licenses/by-nc/4.0/) which permits copy and redistribute the material just in noncommercial usages, provided the original work is properly cited. 
<smiles>CCCC(CCC)C(=O)O</smiles>

Tbrain concentrations GABA by: Enhanced synthesis, Inhibition of Metabolism, Interruption of its reuptake
Inhibition of neuronal firing by prolonging recovery from inactivation of voltage-sensitive sodium channels

Diminish the stream of calcium ions throughout T-type calcium channels, therefore dropping neuronal pacemaker current

Figure 1. Mechanism of Action of Valproic Acid

\section{Results}

Dosage modification should be based on the decision related to prolonged or short-term management [5-7]. All forms of drug such as divalproex sodium, sodium valproate and valproic acid distribute comparable amounts of the dynamic valproate ion. Dissimilarities in the rate of dissolution and following bioavailability of the VPA forms of divalproex sodium necessitate dissimilar overall daily doses of the drug to attain similar steadystate plasma levels of valproic acid. At recommended serum concentrations of $50-100 \mathrm{mg} / \mathrm{mL}$, it is a widely used to treat epilepsy and bipolar disorder. Peak plasma concentrations or Cmax are reached in 1 - 4 hours after oral form of the sodium salt or VPA, in 3 - 5 hours for delayed-release, and in 4-17 hours subsequent to extended-release tablets. More than a few days might be requested to see the complete property of a given prescribed amount. In complex partial seizures, simple and complex absence seizures, the starting dosage of all of the VPA products is $10-15 \mathrm{mg} /$ $\mathrm{kg}$ /day with a maximum dosage of $60 \mathrm{mg} / \mathrm{kg} / \mathrm{day}$ and for migraine prophylaxis is $500-1000 \mathrm{mg} /$ day. For chronic prescription related to bipolar disorders a minimum of $250 \mathrm{mg} /$ day to $3000 \mathrm{mg} /$ day with a vigilant monitoring of drug concentration in blood might be the option for a suitable treatment. For acute treatment of bipolar disorders the minimum dose would be $1000 \mathrm{mg}$ a day.

Table 1 shows the kinetic behavior of this drug after prescription. With a half-life of 9 - 16 hours, it appears that the drug follows a variable absorption pattern with a protein binding (PB) concentration-dependent, from $90 \%$ at $40 \mathrm{mg} / \mathrm{mL}$ to $81.5 \%$ at $130 \mathrm{mg} / \mathrm{mL}$. The pattern of metabolism is $30-50 \%$ and over $40 \%$ glucuronide conjugation and mitochondrial $\beta$-oxidation respectively. It seems hemodialysis could decrease concentration of drug in whole blood by $20 \%$. Therefore patients with hemodialy- sis might need higher doses of drug to control seizure attack. Epileptic convulsions could be apparent by a range of clinical norms. Suitable management should be based on individualized epileptic patient that usually take into explanation related to all the three features of analysis of seizure category and epilepsy pattern, reality of comorbidity interaction between associated drugs. For more than 4 decades VPA stipulate in clinical practice as an anticonvulsant to control seizures in forms of tonic-clonic (grand mal), complex partial and juvenile myoclonic. Published report by International League Against Epilepsy (ILAE) authorizes the efficiency and value of ethosuximide and VPA for children with absence seizures as initial monotherapy [2-12]. Karlov et al. [11] reported a prescription based on valproate in dose $15-25 \mathrm{mg} / \mathrm{kg} /$ day and levetiracetam in dose $20-25 \mathrm{mg} / \mathrm{kg} /$ day that were used in the treatment of 23 patients with juvenile myoclonic, juvenile absence, and convulsive idiopathic generalized and children absence epilepsy. Optimistic modifications in the incidence of convulsions and epileptic action observed in the electroencephalography (EEG) [11].

Valproate is the drug of main option for management in juvenile absence epilepsies (JAE) accompanied by generalized tonic-clonic seizures. If the patient does not appropriately answer, then prescription of lamotrigine or ethosuximide is suggested [13]. However in treatment of cortical myoclonus levetiracetam is first-line treatment but VPA and clonazepam are regularly prescribed. The efficacy of VPA in cortical-subcortical myoclonus has been reported recently by Caviness and Brown [14]. In cortical myoclonus, the most effective drugs are VPA, clonazepam, levetiracetam and piracetam. For corticalsubcortical myoclonus, VPA is the drug of choice. Lamotrigine can be used either alone or in combination with VPA. Leveti- 
racetam or zonisamide can also be used as adjunct therapy with VPA $[14,15]$. The risk of seizures differs between $60 \%$ and $100 \%$ with low grade gliomas and between $40 \%$ and $60 \%$ in glioblastomas. An innovative and thrilling imminent is the likely involvement of VPA to prolonged survival, mainly in glioblastomas [16]. For the treatment of absence epilepsy, it seems that older AEDs such as ethosuximide and VPA are more efficacious than newer AEDs. Due to reduced side effects, ethosuximide remains the first line treatment for childhood absence epilepsy [2-4].

According to expert opinion published recently, VPA might be the most effective drug in idiopathic generalized epilepsies. It should be avoided in women of childbearing age due to its safety profile. In patients with secondary generalized tonic-clonic seizures, AEDs for which the impact on this seizure type has been formally assessed and which have established superior efficiency than placebo might preferentially be used, such as lacosamide, perampanel and topiramate [17]. In juvenile myoclonic epilepsy, lack of response to VPA was noted in $19 \%$ of patients [18]. Corresponding psychiatric disorders demonstrated major association with lack of answer to VPA. The neuroprotective properties of drug have been established in numerous forms of severe central nervous system damages, such as stroke, traumatic brain injury and spinal cord injury. The drug preserves the brain from impairment development via anti-inflammatory, antiapoptotic and neurotrophic effects [19-22]. Elderly are usually more at risk to the adverse effects of VPA than younger adults. Therefore, in this population, the drug should be started at low dosages and titrated gradually. Some of the most troublesome side effects of VPA could be mentioned as: sedation, cognitive side effects and osteoporosis. VPA-drug interactions should be given particular deliberation. It is well known that the pharmacokinetics of all AEDs is altered in the elderly. As renal and metabolic clearance changes in elderly patients, therefore envisaging pharmacokinetic variation seems to be difficult. It seems that there is no agreement on whether VPA should be thought as the ideal first-line management in the elderly [20]. Table 2 shows some of the most clinical properties related to VPA. Due to interand intra-individual variation drug dosing depends on maintenance or acute application in relation to drug concentration monitoring. In combination therapy as a mood stabilizer, lithium might offer a proper synergistic effect. For glutamate excitotoxicity [21], amyotrophic lateral sclerosis [23], Huntington's disease [24], valproate might provide better clinical efficacy. Following severe intake of a large dose of VPA, central nervous system depression, could be categorized from drowsiness to coma. Respiratory depression, although not a common finding, has also been reported. A vigilant dosing of amitriptyline with VPA co medication is recommended since this addition may guide to a significant raise of amitriptyline serum level [2-4]. In combination with carbapenems, monitoring of VPA levels seems to be necessary [25]. Simultaneous prescription with olanzapine significantly decreases serum concentrations of VPA. Smoking also decreases the levels of VPA [26]. The prescription of this drug in pregnancy could increase the risk of spina bifida, cognitive impairments and many other defects by three times. VPA could increase the risk of autism. A high dose of folic acid is recommended for pregnant women. Second trimester ultrasound scans and alpha fetoprotein measurements seem to be necessary. The drug may have an effect on embryonic heart development and may be associated with congenital cardiovascular defects. The drug is an antagonist of folate and could cause neural tube defects that could lead to lower intelligence quotient scale (Iqs) [27-29].

The relationship of $25(\mathrm{OH}) \mathrm{D}_{3}$ levels with anticonvulsant showed that the possibility of vitamin D deficiency could be considered in pediatric patients taking anticonvulsants if they have mental retardation or developmental delay or if they have been taking anticonvulsants for more than 2 years or taking hepatic enzyme inducing drugs [30]. Also there is a significant alteration in trace element status with VPA [31]. A notably small concentration of histamine associates with drug resistance and specifies participation of the $\mathrm{H} 1 / \mathrm{H} 3$ receptors [32]. It seems that overweight patients with epilepsy treated with VPA are at superior possibility of metabolic syndrome than individuals who are just overweight. Therefore, the homeostasis model assessment index should be checked in overweight patients who obtain VPA treatment, rather than observing body weight only [33].

Table 1. Sodium Valproate Pharmacokinetic Data in Epileptic Patients

Pharmacokinetic Data [1-41]

Target trough level (Co): 50 - 100 mg/L, Bioavailability (F): rapid absorption, Half Life (t1/2): 9 - 16 h

Dose (Initially: 10 - 15 mg/kg/day in 1- 3 divided dose) $\rightarrow$ Hemodialysis $\downarrow$ Co by about 20\%. Administered with food to $\downarrow$ adverse gastrointestinal side effects

Protein Binding(PB): Concentration-dependent, from $90 \%$ at $40 \mu \mathrm{g} / \mathrm{mL}$ to $81.5 \%$ at $130 \mu \mathrm{g} / \mathrm{mL}$

Metabolism: Hepatic-glucuronide conjugation 30 - 50\%, mitochondrial $\beta$-oxidation over 40 
Tolou-Ghamari Z et al.

Table 2. The Most Important Clinical Properties of Sodium Valproate

Clinical properties of VPA [1-41]

Side effects $\rightarrow$ Most common: Dyspepsia-Weight gain. Less common: Fatigue, peripheral edema, acne, feeling cold, blurred vision, dizziness, CNS depression ranging from drowsiness to coma, hair loss, headaches, tremors, respiratory depression, marked bone marrow depression, metabolic acidosis, hypoglycemia, hypophosphatemia, hypocalcemia, hypernatremia, hyperammonemia (for treatment use L-carnitine). In pregnancy: Spina bifida, cognitive impairments, other defects

If the dose $\uparrow \rightarrow$ tremor, stupor, respiratory depression, coma, metabolic acidosis, death. In acute poisoning Co $\uparrow \rightarrow 150$ $1500 \mathrm{mg} / \mathrm{L}$. Hemoperfusion or hemofiltration $\rightarrow$ removing the drug from the body

\section{Supplemental L-carnitine is used in this event}

Interactions with: Carbamazepine $\rightarrow$ prolongs the effects of carbamazepine and delaying its excretion. Amitriptyline and nortriptyline $\rightarrow$ valproic acid could decrease the clearance of these drugs. Aspirin $\rightarrow \uparrow$ Co. Clonazepam $\rightarrow$ lead to profound sedation, $\uparrow$ the risk of absence seizures. Lamotrigine $\rightarrow \downarrow$ the clearance of lamotrigine, therefore dose of lamotrigine $\downarrow$ half meropenem $\rightarrow \downarrow \mathrm{C}_{0}$; Valproic acid $\rightarrow \downarrow$ Co of olanzapine, $\downarrow$ clearance of clonazepam $\rightarrow$ sedation and $\uparrow$ the risk of absence seizures

Indications $\rightarrow$ Epilepsy, glutamate excitotoxicity, amyotrophic lateral sclerosis, Huntington's disease

Contraindications $\rightarrow$ Pregnancy, severe liver or renal damage, metastatic cancer, pancreatitis, human immunodeficiency virus, depression of bone marrow, coagulation hematological disorders

\section{Discussion}

Several forms of epilepsy seem to be the most severe brain complaints that prevailing seizure, autonomic movement, reduced awareness and many other symptoms [33]. Related to the prevalence of epilepsy it seems that it might be affected many people (more than 40 million) of all ages worldwide [34]. The etiology of epilepsy could be an imperative crucial feature in its management and prediction. Analytical and curative progresses recommend that the fundamental division, management and prediction of the population with epilepsy may have undergone some variation. A recent publication by American Association of Poison Control Centers, reported 9,069 human ingestions of VPA in 2004 [2-7].

The wide spectrum antiepileptic drug, sodium valproate (VPA) is generally used for different categories of convulsions such as complex partial seizures, simple and complex absence seizures and monotherapy should be acknowledged as the most therapeutic strategies in epileptic population. An abnormal alteration in gene function could be supposed to be concerned in a broad range of human disorder as well as behavioral, cognitive and neurodegenerative pathologies. Therefore assessment of VPA doses seems to be mostly on the patient's history of disease and the category of product that could be linked to pharmacokinetic (PK) parameters. According to a recent report for time to twelve month remission, the multivariable figure included first degree relative with epilepsy, age at randomization, neurological insult, number of tonic-clonic seizures before randomization, seizure type and treatment. The figure for time to twenty-four month remission was similar and also included febrile seizure history. The multidrug resistance protein 2 gene may determine individual susceptibility to adverse drug reactions in the central nervous system by limiting brain access of VPA [2-7, 35-37].

Related to the efficacy of VPA for the long-term management of bipolar disorder, there is inadequate reported data. Polytherapy based on simultaneous use of amitriptyline with VPA needs therapeutic drug monitoring because this combination may lead to an extraordinary increase of amitriptyline serum levels [38]. Concurrent use of VPA and carbapenem antibiotics could cause decrease in the VPA levels. The scope of this reduction seems to be greater in patients with meropenem than imipenem or ertapenem [25].

In patients with severe liver damage, pancreatitis, human immunodeficiency virus depression of bone marrow, coaglation hematological disorders should be contraindicated. Liver, pancreas and hematological dysfunction appear to be common. Weight gain and dyspepsia could be mentioned as one of the main important side effects associated to sodium valproate cause. Fatigue, peripheral edema, acne, feeling cold, tremors, blurred vision hair loss dizziness, and headaches could be mentioned as less common side-effects. Sodium valproate might cause an increase in the level of ammonia in the blood lead to hyperammonemia.

In this circumstance the patient might suffer from vomiting and sluggishness, mental change and brain damage. Even when the blood concentration is reported as normal range, this drug could cause hyperammonemia and encephalopathy. In these patients lactolose could not alleviate the hyperammonemia and L-carnitine is used in toxicity related to valproate. Prescription of activated charcoal and gastric lavage could be useful if carried out within one hour of intake. There is also publication that reported brain encephalopathy without hyperammonemia or elevated levels. However polymorphisms in the 
Tolou-Ghamari Z et al.

genes encoding carbamoyl-phosphate synthase 1 and $\mathrm{N}$ acetylglutamate synthase might be risk factors for the development of hyperammonemia during valproic acidbased therapy but carbamoyl-phosphate synthase polymorphism may not be associated with the development of hyperammonemia in Japanese population [2-7, 39-41].

Concurrent use of VPA with olanzapine significantly decreases serum concentrations of olanzapine. In these patients the higher doses of olanzapine should be considered [26]. Tremor, stupor, respiratory depression, coma, metabolic acidosis and death have been reported related to higher trough levels. VPA increases the amount of carbamazepine-epoxide by disturbing its elimination. The cutoff point forecasting hyperammonemia seems to be VPA dose of $30.4 \mathrm{mg} / \mathrm{kg} /$ day and $\mathrm{VPAC}_{0}$ of $90.9 \mathrm{mg} / \mathrm{mL}$ [1-41].

Finally, in order to highlight the importance of information, for the best individual clinical pharmacotherapy recommendation, VPA treatment strategies in Iranian epileptic population should be accompanied by many factors such as; 1) type of epilepsy, 2) VPA dose and formulation, 3) decision for monotherapy or rational polypharmacy 4) inhibition or induction of CYP450 5) addition of other drugs to VPA 6) an overall prediction of VPA individualized clinical pharmacokinetics.

\section{Acknowledgements}

The authors wish to thank to Isfahan university of medical sciences.

\section{References}

1. Burton BS. On the propyl derivatives and decomposition products of ethylacetoacetate. Am Chem J.1882;3:385-95.

2. Tenney JR, Jain SV. Absence Epilepsy: older vs newer AEDs. Curr Treat Options Neurol. 2014;16(5):290.

3. Cincarova L, Zdrahal Z, Fajkus J. New perspectives of valproic acid in clinical practice. Expert Opin Investig Drugs. 2013;22(12):1535-47.

4. Manoguerra AS, Erdman AR, Woolf AD, Chyka PA, Caravati EM, Scharman EJ, et al. Valproic acid poisoning: an evidence-based consensus guideline for out-of-hospital management. Clin Toxicol (Phila). 2008;46(7):661-76.

5. Ghamari ZT, Habibabadi JM, Palizban AA. Evidence-based pharmacotherapy of epilepsy. Arch Neurosci . 2015;2(2): e18468.

6. Tolou-Ghamari Z. Antiepileptic drugs (AEDs) polypharmacy could lead to buried pharmacokinetic interactions due to CYP450. Drug Metab Lett. 2012;6(3):207-12.

7. Tolou-Ghamari Z, Zare M, Habibabadi JM, Najafi MR. Antiepileptic drugs: a consideration of clinical and biochemical outcome in patients with epilepsy. Int J Prev Med. 2013;4(Suppl 2):S330-7.

8. Lemperiere T. [Brief history of the development of valproate in bipolar disorders]. Encephale. 2001;27(4):365-72.

9. Inoue T, Matsumoto R, Ikeda A. [Treatment of epilepsy in patients of adolescence and adulthood]. Nihon Rinsho. 2014;72(5):853-8.

10. Lin WW, Jiao Z, Wang CL, Wang HY, Ma CL, Huang PF, et al. Population pharmacokinetics of valproic acid in adult Chinese epileptic patients and its application in an individualized dosage regimen. Ther Drug Monit. 2015;37(1):76-83.

11. Karlov VA, Freidkova NV, Rusanova LV. [Complex therapy of idiopathic forms of epilepsy with small doses of valproates and levetiracetam]. Zh Nevrol Psikhiatr Im S S Korsakova. 2012;112(6 Pt 2):37-9.

12. Glauser T, Ben-Menachem E, Bourgeois B, Cnaan A, Guerreiro C, Kalviainen R, et al. Updated ILAE evidence review of antiepileptic drug efficacy and effectiveness as initial monotherapy for epileptic seizures and syndromes. Epilepsia. 2013;54(3):551-63.
13. Lemmon ME, Kossoff EH. New treatment options for lennoxgastaut syndrome. Curr Treat Options Neurol. 2013;15(4):519-28.

14. Caviness JN, Brown P. Myoclonus: current concepts and recent advances. Lancet Neurol. 2004;3(10):598-607.

15. Caviness JN. Treatment of myoclonus. Neurotherapeutics. 2014;11(1):188-200.

16. Vecht CJ, Kerkhof M, Duran-Pena A. Seizure prognosis in brain tumors: new insights and evidence-based management. Oncologist. 2014;19(7):751-9.

17. Rheims S, Ryvlin P. Pharmacotherapy for tonic-clonic seizures Expert Opin Pharmacother. 2014;15(10):1417-26.

18. Jayalakshmi S, Vooturi S, Bana AK, Sailaja S, Somayajula S, Mohandas S. Factors associated with lack of response to valproic acid monotherapy in juvenile myoclonic epilepsy. Seizure. 2014;23(7):527-32.

19. Seo J, Howell MD, Singh NN, Singh RN. Spinal muscular atrophy: an update on therapeutic progress. Biochim Biophys Acta. 2013;1832(12):2180-90.

20. Perucca E, Aldenkamp A, Tallis R, Kramer G. Role of valproate across the ages. Treatment of epilepsy in the elderly. Acta Neurol Scand Suppl. 2006;184:28-37.

21. Lin Y, Lin Y, Li X, Lao G, Jiang Z, Ye B, et al. Monotherapy with lithium or valproate or respectively combination therapy with quetiapine have similar effect on cognitive functions in Chinese euthymic patients with bipolar disorder: a cross-sectional survey. J Clin Psychopharmacol. 2013;33(6):817-9.

22. Shao L, Young LT, Wang JF. Chronic treatment with mood stabilizers lithium and valproate prevents excitotoxicity by inhibiting oxidative stress in rat cerebral cortical cells. Biol Psychiatry. 2005;58(11):879-84

23. Pradat PF, Attarian S, Camdessanche JP. Research in amyotrophic lateral sclerosis: What is new in 2009? Curr Neurol Neurosci Rep. 2011;11(1):67-77.

24. Lauterbach EC. Neuroprotective effects of psychotropic drugs in Huntington's disease. Int J Mol Sci. 2013;14(11):22558-603.

25. Yoon H, Kim DH. Unusual drug reaction between valproate sodium and meropenem. Int J Clin Pharm. 2013;35(3):316-8.

26. Haslemo T, Olsen K, Lunde H, Molden E. Valproic acid significantly lowers serum concentrations of olanzapine-an interaction effect comparable with smoking. Ther Drug Monit. 2012;34(5):512-7.

27. Gandelman-Marton R, Neufeld M. [Epilepsy in pregnancy]. Harefuah. 2013;152(8):473-6.

28. Begum S, Sarma SP, Thomas SV. Malformation in index pregnancy in women with epilepsy is not followed by recurrence in subsequent pregnancy. Epilepsia. 2013;54(12):e163-7.

29. Ahir BK, Pratten MK. Developmental cardiotoxicity effects of four commonly used antiepileptic drugs in embryonic chick heart micromass culture and embryonic stem cell culture systems. Toxicol In Vitro. 2014;28(5):948-60.

30. Baek JH, Seo YH, Kim GH, Kim MK, Eun BL.Vitamin D levels in children and adolescents with antiepileptic drug treatment. Yonsei Med J. 2014;55(2):417-21.

31. Sarangi SC, Tripathi M, Kakkar AK, Gupta YK. Effect of antiepileptic therapy on trace elements status in Indian population in a tertiary care hospital from northern India: a cross sectional study. Epilepsy Res. 2014;108(5):917-27.

32. Singh E, Pillai KK, Mehndiratta MM. Characterization of a lamotrigine - resistant kindled model of epilepsy in mice: evaluation of drug resistance mechanisms. Basic Clin Pharmacol Toxicol. 2014;115(5):373-8.

33. Fang J, Chen S, Tong N, Chen L, An D, Mu J, et al. Metabolic syndrome among Chinese obese patients with epilepsy on sodium valproate. Seizure. 2012;21(8):578-82.

34. Duncan JS, Sander JW, Sisodiya SM, Walker MC. Adult epilepsy. Lancet. 2006;367(9516):1087-100.

35. Depondt C, Shorvon SD. Genetic association studies in epilepsy pharmacogenomics: lessons learnt and potential applications. Pharmacogenomics. 2006;7(5):731-45.

36. Bonnett LJ, Tudur Smith C, Smith D, Williamson PR, Chadwick D, Marson AG. Time to 12-month remission and treatment failure for generalised and unclassified epilepsy. J Neurol Neurosurg Psychiatry. 2014;85(6):603-10. 


\section{Tolou-Ghamari Z et al.}

37. Yi JH, Cho YJ, Kim WJ, Lee MG, Lee JH. Genetic variations of ABCC2 gene associated with adverse drug reactions to valproic acid in Korean epileptic patients. Genomics Inform. 2013;11(4):254-62.

38. Unterecker S, Burger R, Hohage A, Deckert J, Pfuhlmann B. Interaction of valproic acid and amitriptyline: analysis of therapeutic drug monitoring data under naturalistic conditions. J Clin Psy chopharmacol. 2013;33(4):561-4.

39. Park MK, Lim KS, Kim TE, Han HK, Yi SJ, Shin KH, et al. Reduced valproic acid serum concentrations due to drug interactions with carbapenem antibiotics: overview of 6 cases. Ther Drug Monit. 2012;34(5):599-603.

40. Inoue K, Suzuki E, Takahashi T, Yamamoto Y, Yazawa R, Takahashi Y, et al. $4217 \mathrm{C}>\mathrm{A}$ polymorphism in carbamoyl-phosphate synthase 1 gene may not associate with hyperammonemia development during valproic acid-based therapy. Epilepsy Res. 2014;108(6):1046-51.

41. Motamedi M, Sahraian MA, Moshirzadeh S. A cross sectional study evaluating perceived impact of epilepsy on aspects of life. Zahedan J Res Med Sci. 2012;14(4):33-6. 